\begin{tabular}{|l|l|}
\hline $\begin{array}{c}\text { European Association for the } \\
\text { Development of Renewable Energies, Environment } \\
\text { and Power Quality (EA4EPQ) }\end{array}$ & $\begin{array}{l}\text { International Conference on Renewable Energies and Power Quality } \\
\text { (ICREPQ'12) } \\
\text { Santiago de Compostela (Spain), 28th to 30th March, 2012 }\end{array}$ \\
\hline
\end{tabular}

\title{
Seamless disconnection and reconnection transients for Micro-Grids
}

\author{
J. Rocabert ${ }^{1}$, Member, IEEE, A. Luna ${ }^{1}$, Member, IEEE, I. Candela ${ }^{1}$, Member, IEEE, P. Rodriguez ${ }^{1}$, Member, IEEE. \\ ${ }^{1}$ Electrical Engineering Department \\ Technical University of Catalonia \\ (UPC) Terrassa, Spain \\ Contact Author: rocabert@ee.upc.edu
}

\begin{abstract}
.
The increasing penetration of generation power plants based on renewable energies in the electrical networks has boosted the number of systems connected to the grid. In this scenario the micro-grid, providing advanced functionalities to improve the stability and operation of the network, have become very popular. In this paper a control strategy for the micro-grid management is presented. The proposed system improves the performance of micro-grids and its interaction with the main network, or also with other micro-grids, under grid voltage transients. This technique gives rise to a simple and robust control scheme to ensure the appropriate micro-grid disconnection from the main grid. In the following, the control algorithm for the intelligent connection agent will be shown, and the grid synchronization system, as well as the voltage and current control loops will be detailed. Finally simulation results obtained using the MATLAB/Simulink \& Psim platform will be presented and discussed.
\end{abstract}

Keywords: micro-grids, Distributed Power Generation, GridConnection and Island operation, Voltage Source Converters, Intentional Islanding

\section{Introduction}

The increasing penetration of renewable energy sources is giving rise to a challenging scenario in the electrical power systems, where the integration of such energy sources must be combined with an ever increasing demand for electrical energy.

Classical power systems structure is characterized by large energy generators and loads connected by radial transmission systems. Over the last years, the great increase of renewable energy sources, among others, has contributed to change this structure that is moving towards a distributed power generation system, where meshed networks connect multiple low scale producers and consumers.

The integration of Distributed Generation is increasing significantly in the last years, mainly, due to the integration of photovoltaic and wind power plant in medium and low voltage grids. Lately, storage energy systems are being integrated into the electrical grid with the aim of improving stability and quality service. In this respect, the short and long term energy storage units can play a major role in the control and operation of microgrids.

The growth of renewable energy system has launched the connection in parallel of voltage source inverters in small isolated grids of photovoltaic generation, batteries and fuel cells, for example. Actually, the same VSI inverters are used to control of weak networks.

In island grids, is usual the use of renewable sources and other small scale distributed generators equipped with voltage source inverter. Similarly, in Uninterruptible Power Supplies (UPS) applications, parallel redundant inverters are used to increase the reliability of the electrical network. In a near future, a lot of distributed generators with dc-ac converters will feed the utility networks, so a good performance of those under any electric grid station must be guaranteed.

One of the essential issues in the integration of renewable energies in distributed generation systems is the parallelization of voltage source inverters in islanding applications. This issue was initially tackled in applications related to Uninterruptible Power Supply UPS, where a redundant power source had to feed critical loads

Conventional supervisory control algorithms are focused on achieving an instantaneous shut down of any power source affected by critical contingencies in order to prevent any case of islanding situation [1]-[2]. In the case of wind power systems, this philosophy changed some years ago, being the wind farms required to ride through transient faults and support the grid under such adverse conditions [3].

Operation of grid connected photovoltaic and wind power systems in intentional island mode under grid fault conditions might be used for improving both the network stability and the energy supply. In this way, development and improvement of island mode detection algorithms is a essential issue [4]. Moreover, the conventional operational of micro-grids during grid faults will change as well.

There are several references in the literature dealing with connection and disconnection of micro-grids from 
the main electrical network For instance, the implementation of an intelligent and autonomous switch, is presented in [5]. This system is based on monitoring the operating conditions of both the micro-grid and the main network and, depending on the status of the main network, the micro-grid connection or disconnection procedure is triggered. The transition between these two operation modes should be as transparent as possible, from the point of view of the loads, in order to guarantee the maximum possible quality in supplying the loads connected to the micro-grid.

In DG three-phase systems, the use of a Phase-Locked Loop (PLL) based on a synchronous reference frame has become a standard to maintain the DG-inverter synchronized with the grid. As an extension, it is also becoming a standard for the control of the converter master of micro-grid in any of the micro-grid operation modes, and especially in the disconnection and reconnection transients. Until now, the various systems proposed by the phase and amplitude voltage government in the connection and re-synchronization transients are based on phase detection and control. In [6], two synchronization PLL loops are required to achieve disconnection and resynchronization process. One designated to detect the micro-grid phase voltage and the other one to main grid phase. This application can be further simplified, as it has been done in [7], using a single PLL to detect the quadrature grid voltage to synchronize the micro-grid with the main grid voltage. In [8]-[9] the performance of the network synchronization when using a PLL, for phase and amplitude error detection in synchronous reference frame (SRF), is proposed.

In this paper a solution for transient and steady-stage control from grid connected to islanded micro-grids will be proposed. A primary control method is presented to achieve a fast transient and a good operability both in grid connected mode and island mode. This strategy presented in the paper is evaluated through simulations.

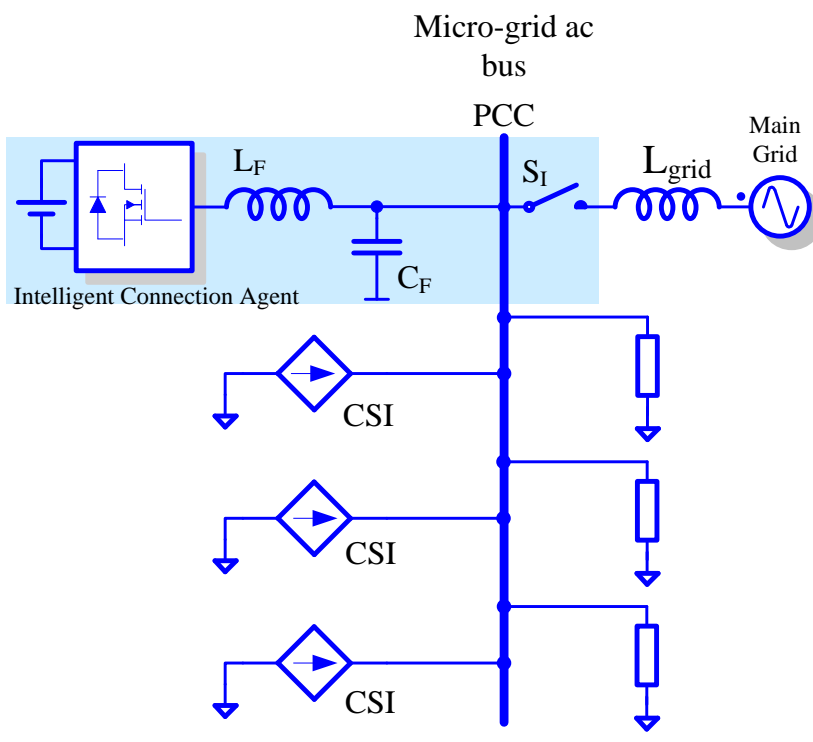

Fig. 1. Equivalent per phase network representation of the micro-grid analised, governed by the intelligent connection agent.

\section{Micro-grid Operation Mode}

In this section the main operation modes of a microgrid, grid connected and islanded mode, are explained. Fig. 1 shows the utility and the micro-grid considered in the purpose of this paper. In this case, the micro-grid consists of different generation units, different loads and a master inverter which control the connection state of the grid switch. As shown in Fig.1, this master inverter or intelligent connection agent (ICA) is formed by a VSI, which takes charge of processing the energy delivered/stored by the micro-grid, and a controlled switch $\left(S_{i}\right)$, which physically connects and disconnects the microgrid to the main grid.

\section{A. Grid Connected Mode}

This will be the usual operating mode, in which the main objective of the ICA is to track the grid voltage. When the micro-grid is working in grid-connected mode, different grid-feeding converters are working to supply the different connected loads, in case of excess or lack of production the grid switch, $S_{\mathrm{I}}$ in Fig. 1, is involved to maintain the power balance and the grid voltage in the Point of Common Coupling (PCC) under the limits.

In this mode, all the loads in the micro-grid are being fed by the main grid or other power sources linked to the micro-grid. Under these operating conditions, in case of having an ICA with a dc bus made by batteries, these would be charged from the grid if necessary.

In the grid connected mode, the ICA-VSI control loop acts as a voltage and frequency follower and also could feeds, or draws, the necessary active and reactive power to collaborate in maintaining the voltage and frequency values within limits set in the grid codes. An example of this application are the so-called grid-supporting inverters, which are designed to flexibly support the voltage and/or frequency of either a stand-alone or a interconnected grid, as shown in [10].

\section{B. Islanding Mode}

When a grid fault occurs, and it is detected, the VSI changes to work in islanding mode and the switch $S_{i}$, in Fig. 1, remains opened. In island operation mode the micro-grid voltage is imposed by a master inverter, connected in the PCC after the grid-switch. This element is usually a VSI supplied from an external dc-source, like a battery or other Energy Storage System (ESS), in gridforming operation mode imposing the voltage amplitude and frequency. The micro-grid VSI comes to act as a grid-forming and the energy is provided from an external dc source, which could be either a battery or another dc source. In this mode, the micro-grid could be composed by other sources, for example, a current source inverter supplied PV panels. In this case, the VSI supplies or stores the necessary amount of energy to maintain the micro-grid energy balance and keep frequency and voltage within the tolerance limits.

In the island mode, the control of the VSI is designed to act as a grid forming element, with the objective of setting the grid voltage amplitude, frequency and phase. The main difference in the control scheme between the island and grid connected modes lies on the fact that in the island 
mode there is not any ac voltage available for starting the VSI voltage reference. Thus, the VSI himself generates the ac voltage of the micro-grid. This voltage is sensed and supplied to the VSI controller as the main feedback signal.

\section{Transient Operating Mode}

Under deficient grid voltage conditions, the main switch $\left(S_{i}\right)$ is turned off and disconnects the micro-grid from the utility. This transient should be as fast as possible in order to ensure that the load on the micro-grid do not experiment any significant transient while the power flow is maintained.

In a similar way to the connection of the islanding mode, another transient state arouses when the micro-grid should reconnect to the network when operating as an island. Before reclosing the main switch $S_{i}$, the ICA should synchronize the phase-angle and the magnitude of the micro-grid voltage with the voltage of the utility network. Once the micro-grid is linked to the main network, the VSI of the ICA changes its operation mode to the grid-connected mode.

\section{Control of the Intelligent Control Agent}

\section{A. Synchronization Loop}

One of the most important aspects to be solved before connecting any generation system to the utility is to perform an accurate synchronization with the network voltage. Most of the grid-tie systems use a synchronization loop based on a PLL, in a SRF, to obtain the phase angle $(\theta)$ from three-phase ac grid voltage [11]. Then, it is used the Clark transformation and Park rotation matrix in direct and quadrature PI-based control. These structures are usually also part of the re-synchronization loop involved in the island to grid-connected transient and vice versa. The method proposed in this paper is composed by an Intelligent Connection Agent (ICA), formed by a voltage source inverter (VSI) and a controlled grid-switch. In this work, a control algorithm based on the adaptation of a Dual Second Order Generalized Integrator based on a Frequency Locked Loop (DSOGI-FLL) [12] has been implemented for controlling the disconnection, resynchronization and reconnection of the micro-grid from the grid. Taking advantage of the frequency detection, performed by the FLL, the synchronization and the current and voltage control loops are implemented in the $\alpha \beta$-stationary reference frame. Thus, the system is less sensitive to transient phase jumps and the controllers are able to deal with balanced and unbalanced grid conditions.

The SRF-PLL presents a proper performance under balanced grids, but its behavior worsens under perturbations and harmonics in the grid voltage. Therefore, the extracted sequence, in presence of grid phase steps or other faults, will not be reliable. A robust process to detect fundamental voltage based on frequency detection systems is implemented to avoid this problems, as is described in [13]-[14]. This technique consists in DSOGI-FLL with an adaptive structure function of the micro-grid operation mode. Fig. 2 shows the structure

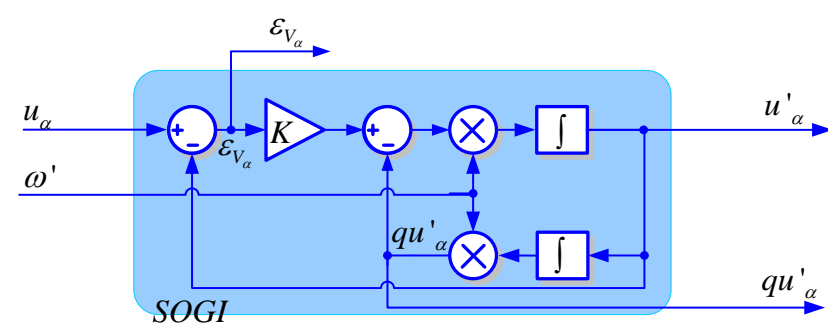

a) Second Order General Integrator structure

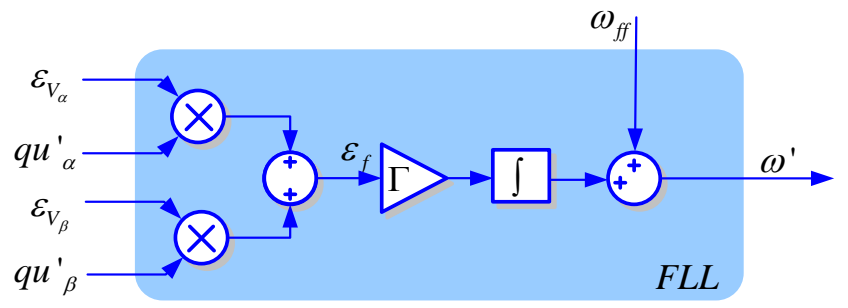

b) Frequency-Locked Loop

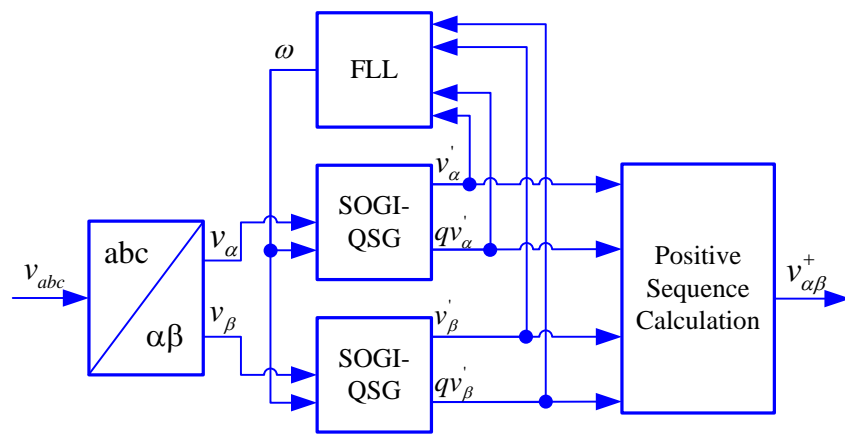

c) Positive Sequence Generation

Fig. 2 Dual SOGI-FLL blocs diagram control with the Positive Sequence Calculation.

used for the grid synchronization in the master inverter, based on the frequency grid detection.

The positive sequence is used to determine the voltage reference for the voltage control loop, in case of the micro-grid is working in island mode. From the oscillating voltage estimated in the synchronization loop, $v_{\alpha \beta}$, the positive sequence is obtained by (1).

$$
v_{\alpha \beta}^{+}=\frac{1}{2}\left[\begin{array}{cc}
1 & -q \\
q & 1
\end{array}\right]\left[\begin{array}{l}
v_{\alpha}^{\prime} \\
v_{\beta}^{\prime}
\end{array}\right]
$$

where $\mathrm{q}$ is a phase-shift time-domain operator lagging $90^{\circ}$ from an original waveform obtained in the Second Order General Integrator - Quadrature Signal Generator (SOGI QSG) for $\alpha$ and $\beta$ voltage component, [15].

\section{B. Current and Voltage Control Loops}

The current and voltage control diagram of the ICA is shown in Fig. 3. As it can be deduced from the figure, the voltage and the current control loops have been implemented using PR controllers working on the $\alpha \beta$ stationary reference frame. An inner current control loop is formed around the filter inductor, and it is designed to have a very fast response. Moreover, an outer voltage control loop is in charge of tracking the grid voltage. The dynamics of this outer voltage loop is slower than the one for the current loop. This grid-follower control works as an active observer of the main grid and it is also responsible of maintaining the voltage reference 
when the ac bus of the micro-grid is disconnected from the utility.

The control system changes as function of the microgrid connection mode. In grid connected mode the current reference $i \alpha \beta^{*}$, is given by a power controller, and the VSI operates as a grid-feeding or grid-supporting converter, injecting the required value of active and reactive power, $\mathrm{P}^{*}$ and $\mathrm{Q}^{*}$. To improve the grid performance and reliability, these power components can be delivered by positive- and negative-sequence currents, like presented in [16]. In island operation mode the ICA operates as a grid-forming converter and gives the required current, $i \alpha \beta^{*}$, to obtain the sinusoidal reference voltage, $v \alpha \beta^{*}$, imposing thus the micro-grid voltage and frequency. The voltage reference, at a fixed frequency $\omega \mathrm{ff}$ $(50 \mathrm{~Hz})$ is generated by the synchronization system (DSOGI-FLL), the Positive-Sequence Calculation block (PSC) and the normalization function.

An inner PR-current controller, implemented on a stationary reference frame has been implemented in order to control the current injection of the VSI with a proper operability in both grid-connected mode and island mode, as is depicted in Fig. 3. The characteristic transfer function of a PR-controller in $\alpha \beta$ reference frame is:

$$
G_{P R}^{\alpha \beta}(s)=K_{p}+\frac{K_{R} s}{s^{2}+\omega^{2}}
$$

where $k_{\mathrm{P}}$ is the proportional gain, $k_{\mathrm{R}}$ is the resonant gain, and $\omega$ is the resonance frequency, which should match up the grid frequency (it is normally given by the DSOGIFLL). The current controller is responsible for injecting the current reference determined by either the voltage loop or the power controller. Anyway, the reference currents can be comprised of positive- and negative-sequence components. The PR controller permits to control positive- and negative-sequence components, while those based on PI in a synchronous reference frame needs to build decoupled loops for controlling both symmetrical components.

From Fig. 3 it can be noticed how the inner control loop is devoted to control the current injection of the VSI, while the outer loop regulates the VSI output voltage. The objective of these control loops is to achieve a fast transient response and a proper operability in both gridconnected and island modes, as well as, under unbalanced conditions. Note that is also used a PR controller in the voltage loop with the same transference function of (2). When the mains is operating in grid-tie mode, the voltage regulation is disabled and the current reference $i_{\alpha \beta}{ }^{*}$, output of the voltage control, is defined by the power controller. Once the mains' fault is detected and the island grid formed, the voltage loop is activated. As a result, the ICA starts operating as a grid-forming converter and gives the required current, $i_{\alpha \beta}{ }^{*}$, to obtain the sinusoidal reference voltage, $v_{\alpha \beta}$, in the ac micro-grid bus. It worth to note that under either voltage imbalance (when in grid connected mode) or load imbalance (when in island mode) the active power delivered by the VSI will have an oscillatory component at twice fundamental frequency. Thus, this oscillation will appear at the dc bus.

\section{Simulation Results}

The performance of the proposed control has been simulated. The VSI, shown in Fig. 1 and Fig. 3, has been simulated with the complete control system. Considering the diagram of Fig. 1, the loads are considered to be passive and with a linear behavior. The simulated system is composed by the utility grid, with the line impedance and an active switch governed by the supervisor control. This switch is used to connect, or disconnect, the microgrid to the main grid. This ac bus is also responsible of feeding the loads. In this case the load is purely resistive.

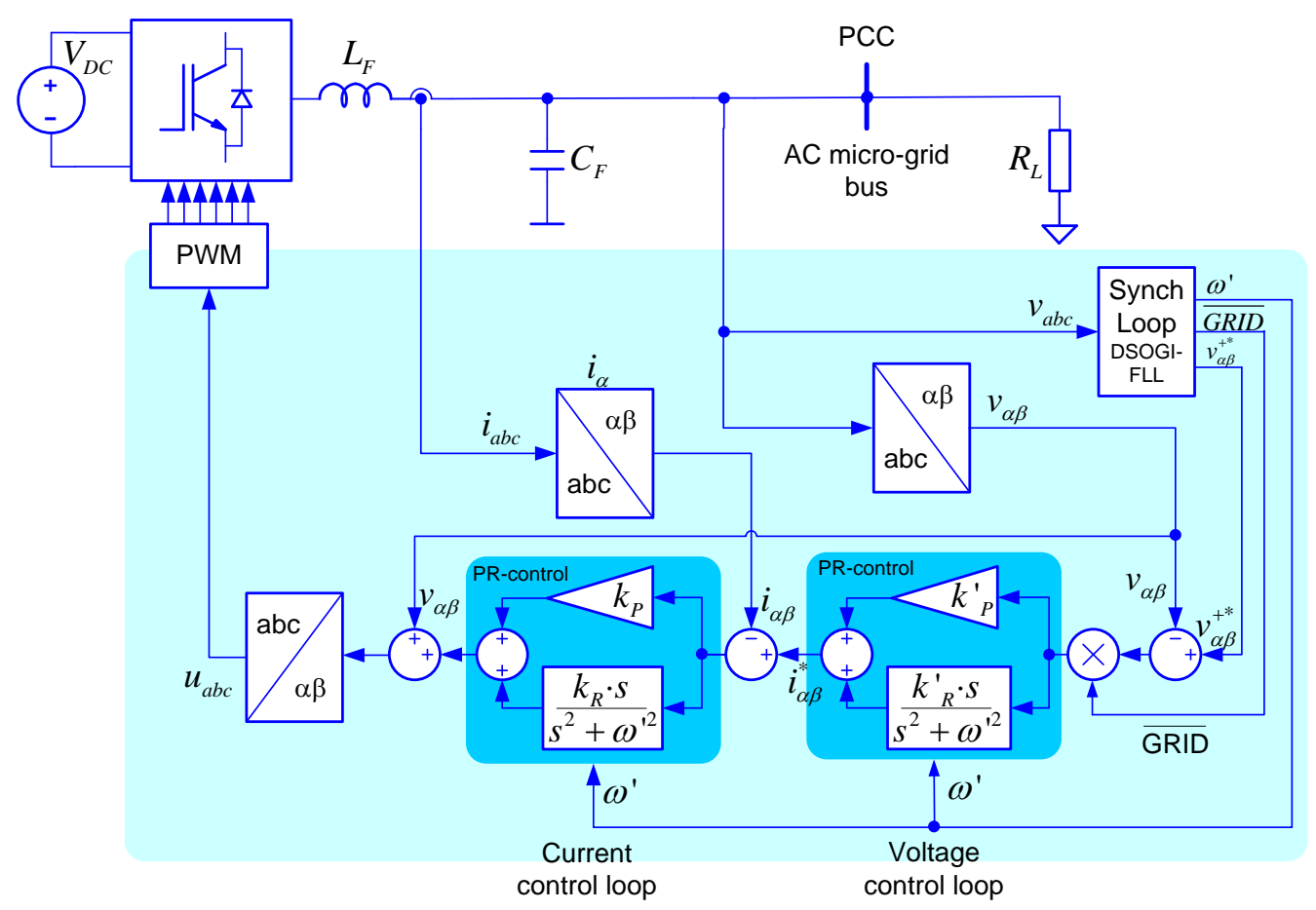

Fig. 3. Current and Voltage control loop applied in the control of the main power source. 
In this simulation the system operates in a gridconnected mode and the load is fully fed by the grid, while the VSI only tracks the grid voltage. Then, at $t=1.03 \mathrm{~s}$, an ideal and symmetrical voltage dip of $95 \%$ is applied at the point of common coupling (PCC), simulating a fault in the utility distribution grid.

The model has been implemented in co-simulation with Simulink/MATLAB and Psim. The environment and the results are shown in the figures below, from Fig 4 to Fig. 5. For the sake of clarity, only one phase of the currents and voltages are shown, although all the simulations had been implemented in three phase systems. The simulation parameters used are given in Table I.

TABLE I

SIMULATION PARAMETERS

\begin{tabular}{lcc}
\hline \hline \multicolumn{1}{c}{ Parameter } & Symbol & Value and Units \\
\hline Filter Inductance & $L_{F}$ & $2.47 \mathrm{mH}$ \\
Filter Capacitance & $C_{F}$ & $20 \mu \mathrm{F}$ \\
dc voltage source & $V_{D C}$ & $700 \mathrm{~V}$ \\
Grid Inductance & $L_{G R I D}$ & $1 \mathrm{mH}$ \\
Grid Resistance & $R_{G R I D}$ & $0.5 \Omega$ \\
Resistive Load & $R_{L O A D}$ & $20 \Omega$ \\
Voltage Loop PR constant values & $k_{P}, k_{R}$ & $0.02,10$ \\
Current Loop PR constant values & $k_{P}, k_{R}$ & 2,10 \\
\hline \hline
\end{tabular}

In Fig 4 and Fig 5. a stable grid-connected mode is observed until the line failure is applied. In a voltage line of $230 \mathrm{~V}_{\mathrm{rms}}$, at PCC a suddenly reduction of $95 \%$ is done. Subsequent to the islanding event and upon its detection, the switch $S_{i}$ is opened and grid-forming control is activated. In Fig. 5 the voltage waveforms that are produced by this fault, $v_{\mathrm{AN}}$, are shown.

The implemented control system includes a voltage normative range detection system, thus when the voltage is out of range the main switch $S_{i}$ is opened and the ICA acts as a grid-forming element, where the frequency and amplitude set point is taken from the values of the voltage detected before the fault.

When a fault occurs, and the out of range detection of the voltage measurement in the micro-grid voltage positive sequence is detected the grid-switch is opened and the synchronization loop starts to generate the reference voltage to the voltage controller. In gridconnected mode, the synchronization loop works with a high dynamic to detect the precise grid frequency to operate in phase with the grid, meanwhile in island mode

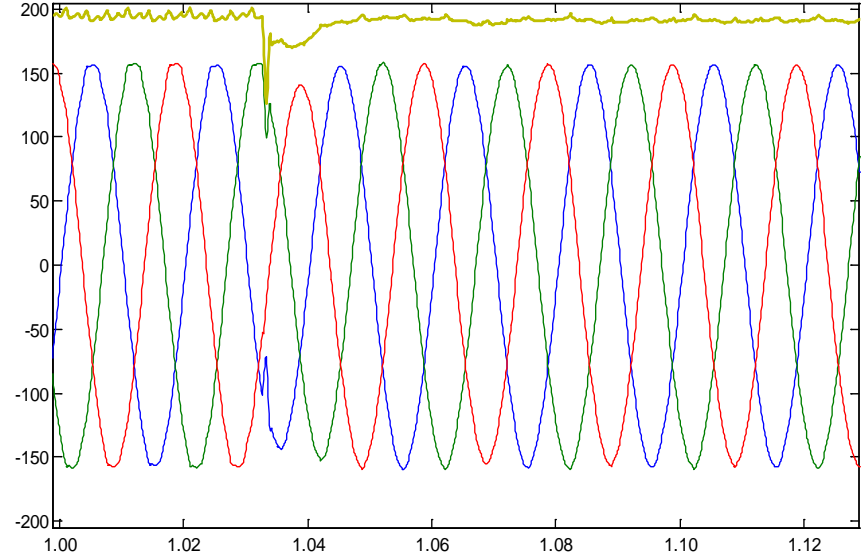

Fig. 4. Micro-Grid voltage waveforms and rms value during a symmetrical grid fault applied on three phase grid voltage. The symmetrical fault occurs at $1.03 \mathrm{~s}$ and is a voltage dip of $95 \%$ of its nominal value.

the frequency estimated by the FLL is fixed to $\omega_{f f}$, so the error is cancelled and $\omega$ remains constant to $\omega_{\text {ref }}$.

In the second part of the simulation, the resynchronization and reconnection process has been evaluated. When the micro-grid is operating in island mode, the main grid is restored again, Fig. 5a. Then, a slow synchronization starts with the main grid frequency, Fig. 5a) - Fig. 5b). Once the resynchronization is achieved the reconnection is done and the grid starts to feed the micro-grid's loads, Fig. 5c).

\section{Conclusion}

This paper proposes a control strategy of a voltage source inverter to operate as a micro-grid intelligent connection agent to form a secure ac bus. The proposed agent has been programmed to control the disconnection or reconnection to the main electrical grid, giving rise to excellent results.

The simulations results, obtained under co-simulation with MATLAB/simulink and Psim, had shown the good performance and a fast dynamics of the proposed system. Also, in grid-connected mode the proposed controller is capable to follow the voltage grid, in amplitude, frequency and phase. In islanding mode, the ICA presents a good regulation feeding the loads with a specific voltage, frequency and phase.

In the transition from a grid-connected mode to an

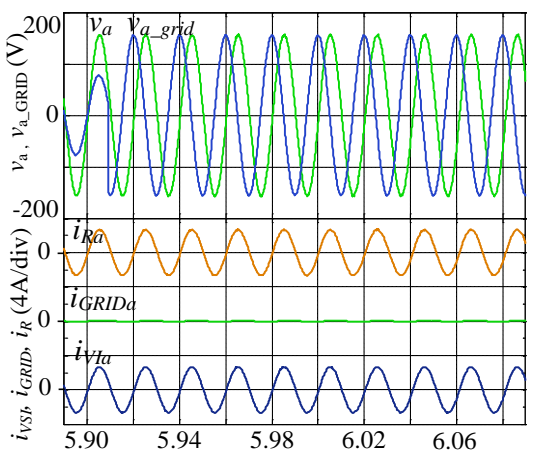

a)

Fig. 5. Micro-Grid voltage waveform during a symmetrical grid fault applied on three voltage grid phase voltage dip of $95 \%$ of its nominal value.
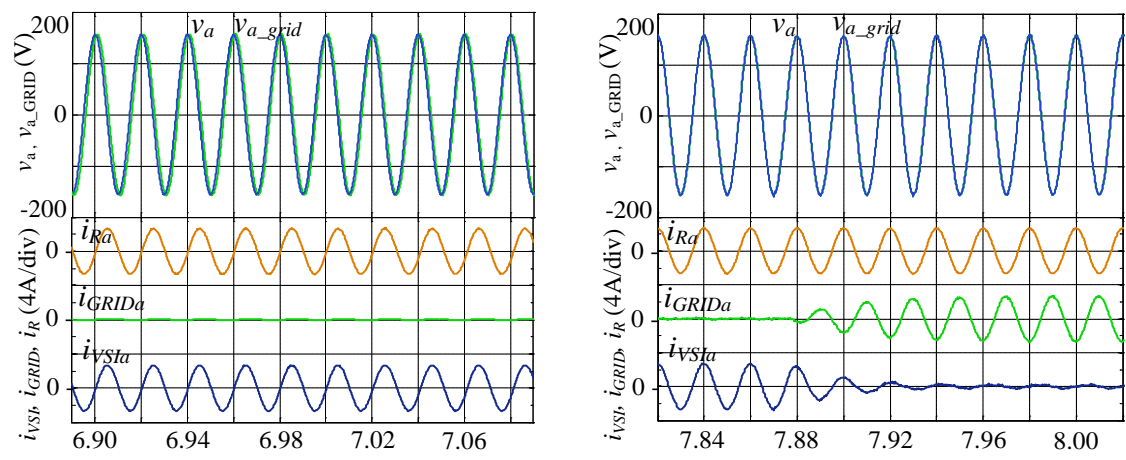

$t(\mathrm{~s}) \mathrm{C})$ $t(\mathrm{~s})$

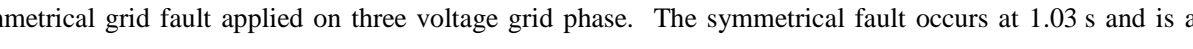


islanded one, a good regulation and a fast dynamic is observed to maintain the setup voltage levels.

\section{Acknowledgment}

This work was supported by the Ministerio de Ciencia y Tecnología, Madrid, Spain, under Grant ENE2008-0841C02-01/ALT.

\section{References}

[1] Z. Ye, A. Kolwalkar, Y. Zhang, P. Du, and R. Walling, "Evaluation of anti-islanding schemes based on nondetection zone concept," IEEE Transactions on Power Electronics, vol. 19, no. 5, pp. 1171-1176, 2004.

[2] M. Liserre, A. Pigazo, A. Dell'Aquila, and V. M. Moreno, "An Anti-Islanding Method for Single-Phase Inverters Based on a Grid Voltage Sensorless Control," IEEE Transactions on Industrial Electronics, vol. 53, no. 5, pp. 14181426, 2006.

[3] J. Matevosyan, T. Ackerman, S. Bolik, and S. Lennart, "Comparison of international regulations for connection of wind turbines to the network," in Nordic wind power conference, 1-2 March 2004.

[4] H. Karimi, A. Yazdani, and R. Iravani, "NegativeSequence Current Injection for Fast Islanding Detection of a Distributed Resource Unit," Power Electronics, IEEE Transactions on, vol. 23, no. 1, pp. 298-307, 2008.

[5] J. M. Guerrero, N. Berbel, J. Matas, J. L. Sosa, and L. G. de Vicuna, "Control of Line-Interactive UPS Connected in Parallel Forming a Microgrid," in Proc. IEEE International Symposium on Industrial Electronics, 2007, pp. 2667-2672.

[6] T. Thacker, F. Wang, and D. Boroyevich, "Islanding Control of a Distributed Generation Unit's Power Conversion System to the Electric Utility Grid," in Proc. IEEE 36th Power Electronics Specialists Conference, 2005, pp. 210-216.

[7] H. Kim, T. Yu, and S. Choi, "Indirect Current Control Algorithm for Utility Interactive Inverters in Distributed Generation Systems," IEEE Transactions on Power Electronics, vol. 23, no. 3, pp. 1342-1347, May 20082008.

[8] Y. Li, D. M. Vilathgamuwa, and P. C. Loh, "Design, analysis, and real-time testing of a controller for multibus microgrid system," IEEE Transactions on Power Electronics, vol. 19, no. 5, pp. 1195-1204, 2004.

[9] R. Teodorescu and F. Blaabjerg, "Flexible control of small wind turbines with grid failure detection operating in stand-alone and grid-connected mode," IEEE Transactions on Power Electronics, vol. 19, no.5, no. 5, pp. 1323-1332, Sep.2004 2004.

[10] D. Georgakis, S. Papathanassiou, N. Hatziargyriou, A. Engler, and C. Hardt, "Operation of a prototype microgrid system based on micro-sources quipped with fast-acting power electronics interfaces," in IEEE 35th Annual Power Electronics Specialists Conference, 2004, pp. 2521-2526.

[11] A. Timbus, R. Teodorescu, F. Blaabjerg, and M. Liserre, "Synchronization Methods for Three Phase Distributed Power Generation Systems. An Overview and Evaluation," in Proc. IEEE 36th Power Electronics Specialists Conference, 2005, pp. 2474-2481.

[12] P. Rodriguez, A. Luna, I. Candela, R. Rosas, R Teodorescu, and F. Blaabjerg, "Multi-Resonant FrequencyLocked Loop for Grid Synchronization of Power Converters Under Distorted Grid Conditions," IEEE Transactions on Industrial Electronics, vol. 58, no. 1, pp. 127-138, 2010.

[13] P. Rodriguez, A. Luna, I. Candela, R. Teodorescu, and F. Blaabjerg, "Grid synchronization of power converters using multiple second order generalized integrators," in IEEE 34th Annual Conference of IEEE Industrial Electronics, 2008, pp. 755-760.

[14] V. M. Moreno, M. Liserre, A. Pigazo, and A. Dell'Aquila, "A Comparative Analysis of Real-Time Algorithms for Power Signal Decomposition in Multiple Synchronous Reference Frames," IEEE Transactions on Power Electronics, vol. 22, no. 4, pp. 1280-1289, 20072007.

[15] P. Rodriguez, A. Luna, M. Ciobotaru, R. Teodorescu, and F. Blaabjerg, "Advanced Grid Synchronization System for Power Converters under Unbalanced and Distorted Operating Conditions," in Proc. IEEE 32nd Annual Conference on IEEE Industrial Electronics, 2006, pp. 5173-5178.

[16] P. Rodriguez, A. Luna, G. Medeiros, R. Tedorescu, and F. Blaabjerg, "Control of STATCOM in wind power plants based on induction generators during asymmetrical grid faults," in Power Electronics Conference (IPEC), 2010 International, 2010, pp. 2066-2073. 